\title{
Tumor deposits are encountered in advanced colorectal cancer and other adenocarcinomas: an expanded classification with implications for colorectal cancer staging system including a unifying concept of in-transit metastases
}

\author{
Giacomo Puppa ${ }^{1,2}$, Hideki Ueno ${ }^{3}$, Masato Kayahara ${ }^{4}$, Paola Capelli ${ }^{5}$, Vincenzo Canzonieri ${ }^{6}$, \\ Romano Colombari ${ }^{1}$, Patrick Maisonneuve ${ }^{7}$ and Giuseppe Pelosi ${ }^{8,9}$ \\ ${ }^{1}$ Division of Pathology, 'G. Fracastoro' City Hospital, Verona, Italy; ${ }^{2}$ PhD Programme in Experimental \\ Medicine and Oncology, University of Insubria, Varese, Italy; ${ }^{3}$ Department of Surgery, National Defence \\ Medical College, Saitama, Japan; ${ }^{4}$ Division of Cancer Medicine, Department of Gastroenterological Surgery, \\ Graduate School of Medical Science, Kanazawa University, Kanazawa, Japan; ${ }^{5}$ Department of Pathology, \\ University of Verona, Verona, Italy; ${ }^{6}$ Division of Pathology, CRO-National Cancer Institute, Aviano, Italy; \\ ${ }^{7}$ Division of Epidemiology and Biostatistics, European Institute of Oncology, Milan, Italy; ${ }^{8}$ Diagnostic \\ Histopathology Unit, European Institute of Oncology, Milan, Italy and ${ }^{9}$ University of Milan School of \\ Medicine, Milan, Italy
}

\begin{abstract}
The seventh edition of the TNM Classification of Malignant Tumors is due to be published soon. In the current version dating back to 2002, tumor deposits, which are metastatic lesions commonly encountered in the routine histopathological examination of advanced colorectal cancer specimens, are classified according to their shape with different implications for staging. So distinguished, these lesions are considered either as metastatic lymph nodes ( $\mathrm{N}$ category) or as vascular invasions ( $\mathrm{T}$ category). We recently proposed a more comprehensive classification approach that also includes the $M$ category. Relying on two of our independent recent studies, we aim here to provide suggestions for a novel classification of tumor deposits with diverse implications for TNM staging system of colorectal cancer. Furthermore, we show that tumor deposits are not limited to colorectal cancer, but they are common to different adenocarcinoma types.
\end{abstract}

Modern Pathology (2009) 22, 410-415; doi:10.1038/modpathol.2008.198; published online 9 January 2009

Keywords: tumor deposits; colorectal cancer; staging; TNM Classification

The current staging system for colorectal cancer is based mainly upon the assessment of the anatomic extent of the disease at the time of diagnosis. ${ }^{1}$ Accordingly, the prognostic heterogeneity of stage III disease ${ }^{2}$ was addressed in the last TNM edition by stratifying patients into three substages (IIIA-C), adopting criteria based on the depth of the intestinal wall involvement and the number of metastatic lymph nodes. ${ }^{3}$ One of the main objectives of the TNM classification, however, is to provide prognos-

Correspondence: Dr G Puppa, MD, Division of Pathology, 'G. Fracastoro’ City Hospital, Via Circnvallazione, 1, San Bonifacio (VR), Verona I-37047, Italy.

E-mail: gpuppa@yahoo.com

Received 4 August 2008; revised 12 November 2008; accepted 19 November 2008; published online 9 January 2009 tic information useful for deciding the best treatment options for the patients, ${ }^{1}$ stratifying them into groups that are prognostically and therapeutically similar. $^{4}$

As a consistent fraction of patients with locally advanced colonic carcinoma experience a relapse, ${ }^{5,6}$ one of the reasons accounting for this failure may be the difficulty in correctly stratifying patient groups with locally advanced disease in different risk categories. Missing additional features may account for remarkable prognostic differences between patients in the same risk category.

Tumor deposits separate from primaries, encountered in the pathology examination of locally advanced colorectal cancer, were previously classified on the basis of their dimension ${ }^{7}$ and currently on the basis of their shape. ${ }^{1}$ In particular, the present 
Stages III and IV

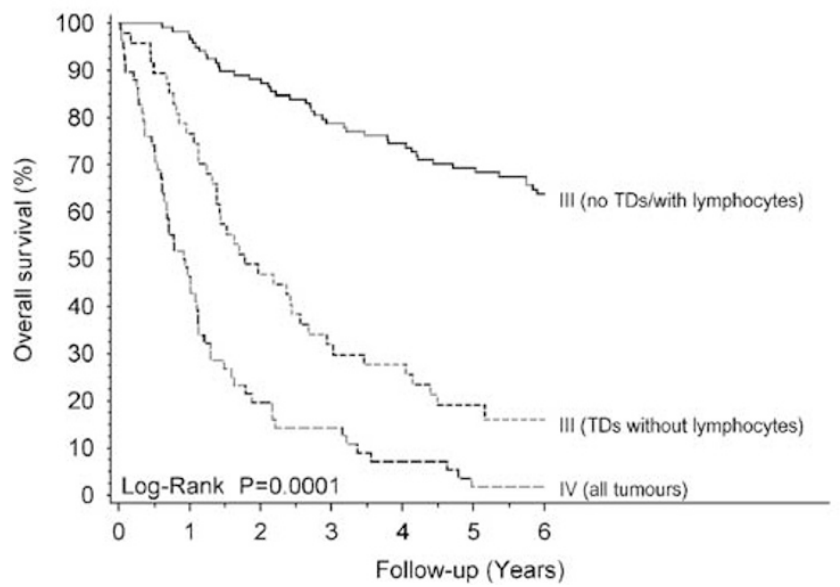

Stage IV

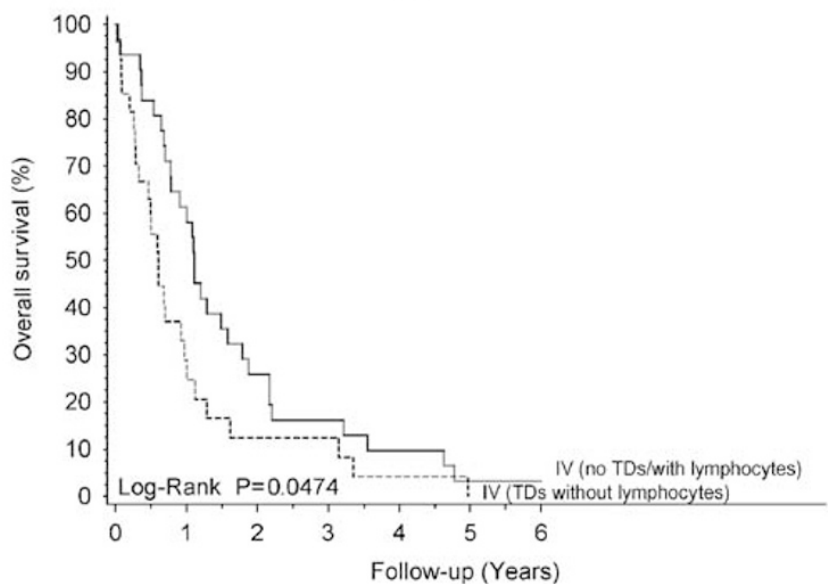

Figure 1 Overall survival of patients with stages III and IV colon cancer according to the absence or presence of tumor deposits without lymphocytes. Survival curves were drawn using the Kaplan-Meier method and comparison between groups was assessed by the log-rank test. Details on population study and tumor characteristics are given in Puppa et al. ${ }^{8}$

TNM classification considers a tumor nodule in the pericolic/perirectal adipose tissue as venous invasion if the nodule has an irregular contour and as regional lymph node metastasis if the nodule has the form and smooth contour of a lymph node. ${ }^{1}$

The shape of tumor deposits, which has become a unique diagnostic clue, is, however, not sufficient to consistently distinguish different types of tumor involvement of the perivisceral fat. ${ }^{8}$

As far as vascular invasion is concerned, it is still unclear whether different types of vascular invasion (lymphatic or venous) are prognostically relevant as independent factors. ${ }^{9}$

A proposal to include venous invasion among stage influencing factors in colorectal cancer has already been made ${ }^{10-12}$ and it is still being debated as to whether this finding per se may have clinical implications on tumor staging. ${ }^{13}$ In this regard, we believe that grouping tumor deposits with other types of vascular invasion will account for the prognostic heterogeneity of these particular lesions.

Studies that considered as tumor deposits all those metastatic occurrences not associated with lymph node involvement showed that these lesions, when grouped as a whole, have a prognosis similar to that of stage III disease, ${ }^{14,15}$ thereby failing to provide an evidence basis to substantiate its inclusion in the TNM system. ${ }^{16}$

Results of a recent meta-analysis based on survival involving 3714 patients, although confirming the consistent adverse prognostic value of tumor deposits, illustrated differences in the hazard ratios between the different studies. ${ }^{16}$

\section{Towards a comprehensive classification and a prognostic staging}

Recently, we proposed a new approach to the classification of tumor deposits, based on a combination of different pathologic features, namely the shape, the morphologic appearance, ${ }^{8,17}$ the presence of lymphoid tissue not organized in the residual lymph node structures ${ }^{8}$ and the degree of involved preexistent anatomical structures, including lymphatic vessels, veins and nerves. ${ }^{8,17}$

A direct implication of our findings is that tumor deposits make up a heterogeneous group of lesions that affects the patients' prognosis and survival differently.

The combination of the shape criterium and the patterns of involvement of preexistent anatomical structures $^{8,17}$ allowed to identify basically three types of tumor deposits.

(1) Tumor deposits confined to a vascular (lymphatic or venous vessel) space ('vascular invasion type, ${ }^{17}$ ); based on the COX proportional regression analysis, the hazard ratio of such lesions was 2.5 and using the Akaike Information Criteria, the value of prognostic staging was greatest when this type was treated as a T factor. ${ }^{17}$

This categorization is in accord with the current TNM staging system where $\mathrm{V}$ and $\mathrm{L}$ are descriptors of T category. ${ }^{1}$

(2) Tumor deposits other than vascular invasion type ${ }^{17}$ viz with no evident association with veins and nerves. These lesions are the most intriguing as they may represent the end product of a variety of processes $^{16}$ therefore their nature is uncertain, thus averting a correct classification.

We believe that an enhanced pathologic analysis in these selected cases is worth it, as the examination of multiple slides has been proved to assess the actual nature of tumor deposits in many cases. ${ }^{18,19}$

In this frame of mind, when a tumor deposit is near to the intestinal wall, this technique may even exclude the presence of a tumor deposit itself, suggesting a continuous growth instead, ${ }^{16}$ thereby assigning the $\mathrm{T}$ category; in other cases some remnants of preexisting structures may show off, allowing a correct classification. 

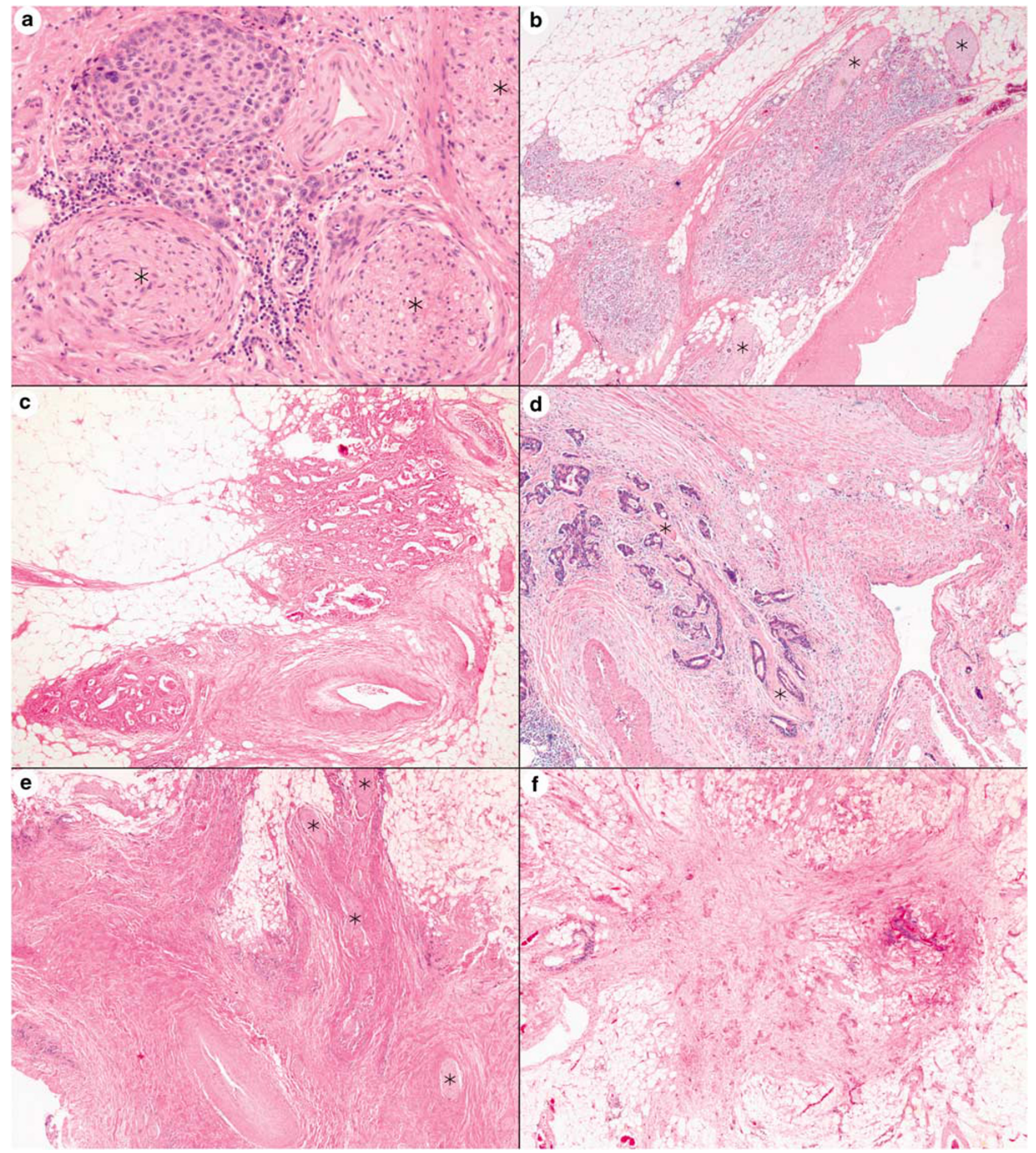

Figure 2 (a) An in-transit metastasis from melanoma; (b) a tumor deposit from gastric carcinoma; (c) a tumor deposit from colorectal cancer in close association with large veins; (d) the core of another tumor deposit from colorectal cancer showing perineural invasion, surrounded by large veins; (e) tumor deposit from extrahepatic colangiocarcinoma, sclerosing type; (f) a tumor deposit from gallbladder adenocarcinoma. Nerves are indicated by asterisks.

However, when the nature of such tumor deposits remain unknown, despite an enhanced pathologic analysis, or when this workload is not performed, the suitable category is the $\mathrm{N}$ factor as using the Akaike Information Criteria, the value of prognostic staging was greatest when these lesions were treated as $\mathrm{N}$ factors. ${ }^{17}$ Accordingly patients with such particular lesions ('extranodal cancer deposits nonvascular invasion-type ${ }^{17}$ ) showed an hazard ratio of 4.7 that is more similar to the nodal involvement 


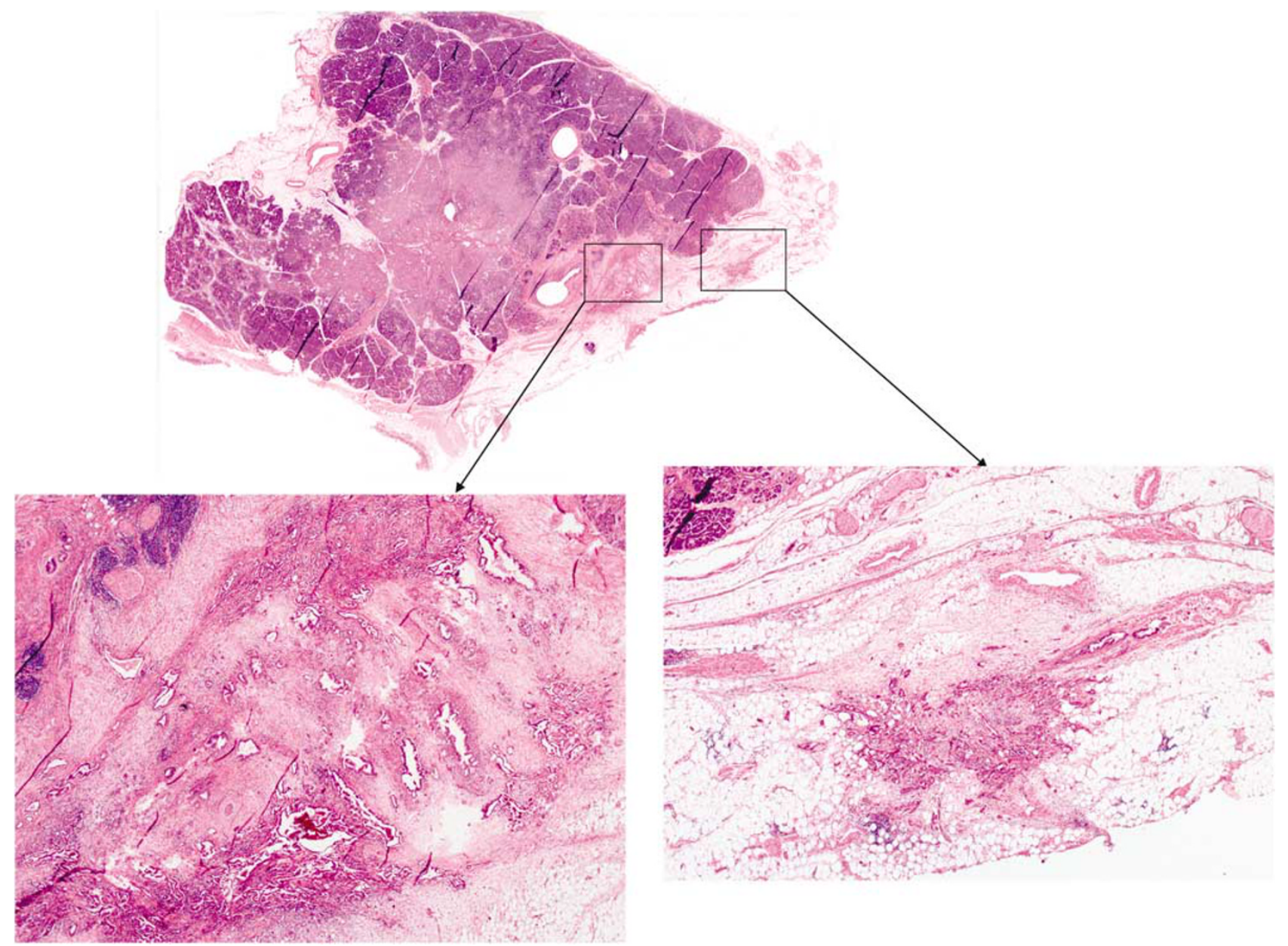

Figure 3 Top whole mount macrosection of pancreaticoduodenectomy showing the primary tumor (on the left, expanded in the inferior left panel) and a tumor deposit, in close association with a vessel (on the right, expanded in the inferior right panel).

(hazard ratio 3.6) than the 'aggressive extranodal cancer deposits' (tumor deposits accompanied by venous and/neural invasion, hazard ratio 8$).{ }^{17}$

Some of these tumor deposits not associated with veins and nerves are surrounded by lymphatic tissue instead: the presence of such lymphoid tissue featuring 'pericolonic tumor deposits with lymphocytes' ${ }^{8}$ correlated with lymphatic invasion and with lymph node metastases: ${ }^{8}$ these particular lesions exhibited a less adverse impact on survival in comparison with tumor deposits involving venous vessels or nerves that instead closely correlated with distant metastases. ${ }^{8}$

This analysis ${ }^{8}$ endorses the proposed categorization. ${ }^{17}$

(3) Tumor deposits extramural venous and perineural invasion type: these lesions, likewise to the ones originally described by Goldstein and Turner, ${ }^{18}$ present an irregular shape, an infiltrative appearance, ${ }^{8,18}$ are not surrounded by lymphocytes ('tumor deposits without lymphocytes ${ }^{8}$ ) and typically are in close association with large vessels and/or nerves ${ }^{8,17,18}$ thereby their strikingly ominous appearance ('aggressive extranodal cancer deposits'17).
These lesions were associated with a very poor clinical outcome ${ }^{8,17}$ with an impact on survival that is greater than lymph node metastases and other types of vascular invasion. ${ }^{8,17}$

We also showed the importance of other features of tumor deposits other than their shape and the types of involvement of preexistent anatomical structures associated with, ${ }^{8,17}$ in particular their dimension $^{8,17}$ and their number, ${ }^{8}$ as found in another study. ${ }^{18}$

Therefore, we tested the prognostic significance of these subtypes of tumor deposits ('tumor deposits without lymphocytes') in stage III according to their presence or absence, in comparison to stage IV.

First, we considered stage III as a whole, as we had already demonstrated that tumor deposits without lymphocytes were not related to lymph node involvement. $^{8}$ Patients with stage III colon cancer with 'tumor deposits without lymphocytes' had an outcome that was definitively poorer than those with stage III colon cancer with no tumor deposits or with tumor deposits with lymphocytes $(P<0.0001)$, more similar to that of patients with stage IV colon cancer (Figure 1 left), although significantly 
different $(P=0.0001$; Figure 1 left). Among patients with stage IV colon cancer, the presence of 'tumor deposits without lymphocytes' was associated with poorer survival (Figure 1 right), as also found in another study. ${ }^{20}$

\section{Tumor deposits are encountered in other adenocarcinoma types and look like meta- static in-transit melanoma}

In our experience of dealing with several types of solid-organ cancers, we realized that tumor deposits are not only limited to colorectal cancer, but also are common to different tumor types, thus reflecting a more general mechanism of invasion through preexisting anatomical structures.

Traditionally, in-transit metastases have been regarded as recurrent disease, resulting from intralymphatic tumor dissemination that is found in the dermis or subcutaneous tissue between the primary melanoma and the regional lymph node basin (Figure 2a). This pattern of recurrence is considered unique and has a reported incidence of $5-10 \% .{ }^{21}$ Although impairment of lymphatic drainage from the primary tumor for whatever reason could predispose to in-transit metastases, it seems more likely that they are the result of an inherently adverse biology of primary tumors, ${ }^{22}$ as was the case in our series of colorectal cancers in which tumor deposits were consistent predictors of a poor prognosis.

When dealing with the routine histological examination of different locally advanced-stage tumor types other than colorectal cancer (Figure 2c and d), including gastric (Figure 2b), biliary duct (colangiocarcinoma: Figure 2e and gallbladder: Figure 2f) and pancreatic (Figure 3) carcinomas, we are faced in some cases with nodular lesions in the perivisceral adipose tissue that strictly resemble the tumor deposits found in colorectal cancer.

\section{Conclusion: suggestion for placing these lesions in an appropriate category in the TNM staging system}

We would encourage describing these findings separately in the pathology report, in particular specifying if some remnants of preexistent anatomical structures are identifiable.

As the new categorization assigns tumor deposits confined to a vascular (lymphatic or venous vessel) space ('vascular invasion type') to T category and tumor deposits other than vascular invasion, not associated with veins and nerves (non-vascular invasion type) to $\mathrm{N}$ category, ${ }^{17}$ the last type of tumor deposits ('tumor deposits without lymphocytes'8 and 'aggressive extranodal cancer deposits' ${ }^{17}$ ) remains to be classified.

We consider these types of tumor deposits as actively growing metastatic lesions spreading along vascular structures and nerves, also found in aggressive neoplasms other than colorectal cancer that would be more appropriately placed in a specific category other than $\mathrm{T}$ and $\mathrm{N}$.

We proposed that these lesions be included in the $\mathrm{M}$ category for staging purposes as they represent in-transit metastases from colorectal cancer. ${ }^{8}$ This approach stands at sharp variance with previous proposals, in which tumor deposits were assigned to either the ' $\mathrm{T}$ ' or ' $\mathrm{N}$ ' factor, inasmuch as in either event, stage grouping would not be influenced. Their presence actually is associated almost exclusively with locally advanced disease thereby removing the potential for upstaging stage II to stage III. ${ }^{16}$

In this frame of mind, a unified conceptual model of tumor deposits such as in-transit metastases may be much more suitable than the TNM 'descriptors' such as lymphatic invasion, venous invasion and neural invasion. In particular, tumor deposits in close association with vessels and nerves herald a high risk for local and systemic recurrence, thereby constituting the true 'trait d'union' between primaries and distant (visceral) metastases.

Our suggestion for the TNM staging system of colorectal cancer is to classify tumor deposits without lymphocytes and associated with veins and/or nerves in the M1a category, whereas cases with distant (visceral) metastases should be designated to the M1b category, likewise to melanoma staging. ${ }^{1}$

\section{Acknowledgement}

We thank Mrs Anna Maria Colussi for her assistance with editing.

\section{References}

1 Sobin LH, Wittekind Ch, (eds). TNM Classification of Malignant Tumors, 6th edn. Wiley-Liss: New York, 2002.

2 Merkel S, Mansmann U, Papadopoulos T, et al. The prognostic in homogeneity of colorectal carcinomas Stage III: a proposal for subdivision of Stage III. Cancer 2001;92:2754-2759.

3 Greene FL, Stewart AK, Norton HJ. A new TNM staging strategy for node-positive (stage III) colon cancer: an analysis of 50042 patients. Ann Surg 2002;236:416-421.

4 Greene FL, Sobin LH. The staging of cancer: a retrospective and prospective appraisal. CA Cancer J Clin 2008;58:180-190.

5 Obrand DI, Gordon PH. Incidence and patterns of recurrence following curative resection for colorectal carcinoma. Dis Colon Rectum 1997;40:15-24.

6 Gramont A. Adjuvant therapy of stage II and III colon cancer. Semin Oncol 2005;32:11-14.

7 Sobin LH, Wittekind Ch, (eds). TNM Classification of Malignant Tumors, 5th edn. Wiley-Liss: New York, 1997.

8 Puppa G, Maisonneuve P, Sonzogni A, et al. Pathological assessment of pericolonic tumor deposits in advanced colonic carcinoma: relevance to prognosis and tumor staging. Mod Pathol 2007;20:843-855. 
9 Compton CC, Greene FL. The staging of colorectal cancer: 2004 and beyond. CA Cancer J Clin 2004;54:295-308.

10 Koukourakis MI, Giatromanolaki A, Sivridis E, et al. Inclusion of vasculature-related variables in the dukes staging system of colon cancer. Clin Cancer Res 2005;11:8653-8660.

11 Sternberg A, Sibirsky O, Cohen D, et al. Validation of a new classification system for curatively resected colorectal adenocarcinoma. Cancer 1999;86:782-792.

12 Sternberg A, Sibirsky O, Cohen D, et al. New approach to the substaging of node-positive colorectal adenocarcinoma. Ann Surg Oncol 1999;6:161-165.

13 Greene FL. Current TNM staging of colorectal cancer. Lancet Oncol 2007;8:572-573.

14 Tateishi S, Arima S, Futami K, et al. A clinicopathological investigation of 'tumor nodules' in colorectal cancer. Surg Today 2005;35:377-384.

15 Lo DS, Pollett A, Siu LL, et al. Prognostic significance of mesenteric tumor nodules in patients with stage III colorectal cancer. Cancer 2008;112:50-54.

16 Nagtegaal ID, Quirke P. Colorectal tumour deposits in the mesorectum and pericolon: a critical review. Histopathology 2007;51:141-149.
17 Ueno H, Mochizuki H, Hashiguchi Y, et al. Extramural cancer deposits without nodal structure in colorectal cancer: optimal categorization for prognostic staging. Am J Clin Pathol 2007;127:287-294.

18 Goldstein NS, Turner JR. Pericolonic tumor deposits in patients with $\mathrm{T} 3 \mathrm{~N}+\mathrm{MO}$ colon adenocarcinomas: markers of reduced disease free survival and intraabdominal metastases and their implications for TNM classification. Cancer 2000;88:2228-2238.

19 Stiles TE, Dean P, Harrison J. Nature and significance of nodular metastases of colonic carcinoma in pericolonic fat [abstract]. Mod Pathol 1995;8:69A.

20 Ishikawa $\mathrm{K}$, Hashiguchi $\mathrm{Y}$, Mochizuki $\mathrm{H}$, et al. Extranodal cancer deposit at the primary tumor site and the number of pulmonary lesions are useful prognostic factors after surgery for colorectal lung metastases. Dis Colon Rectum 2003;46:629-636.

21 Pawlik TM, Ross MI, Johnson MM, et al. Predictors and natural history of in-transit melanoma after sentinel lymphadenectomy. Ann Surg Oncol 2005;12:587-596.

22 Pawlik TM, Ross MI, Thompson JF, et al. The risk of intransit melanoma metastasis depends on tumor biology and not the surgical approach to regional lymph nodes. J Clin Oncol 2005;23:4588-4590. 